\title{
Biometric Indicators of Anterior Segment Parameters Before and After Laser Peripheral Iridotomy by Swept-Source Optical Coherent Tomography
}

Bo Yu

Tianjin Medical University Eye Hospital

Kang Wang

Binzhou Medical University Hospital

Xiaoli Xing ( $\nabla$ xxing04@tmu.edu.cn)

Tianjin Medical University Eye Hospital

\section{Research Article}

Keywords: primary angle closure, laser peripheral iridotomy, optical coherent tomography, anterior segment parameters

Posted Date: December 1st, 2021

DOI: https://doi.org/10.21203/rs.3.rs-1113315/v1

License: (c) (1) This work is licensed under a Creative Commons Attribution 4.0 International License.

Read Full License 


\section{Abstract \\ Background}

Primary angle closure glaucoma (PACG) is the most common type of glaucoma in China. Laser peripheral iridotomy (LPI) is the primary choice to treat PAC. We aim to evaluate the changes and to find the biometric indicators of anterior segment parameters before and after laser peripheral iridotomy (LPI) in primary angle closure (PAC) eyes using swept-source optical coherent tomography (OCT).

\section{Methods}

LPI was performed in 52 PAC eyes of 28 participants. The change of intraocular pressure and anterior segment parameters, including angle opening distance (AOD500), AOD500 area, trabecular iris space area (TISA500), TISA500 volume, trabecular iris angle (TIA500), iridotrabecular contact (ITC) index, ITC area, anterior chamber volume (ACV), anterior chamber depth (ACD), lens vault (LV) and lens thickness (LT) before and 1 week after LPI were measured by Tomey CASIA2 anterior segment OCT. We also estimate and analyze potential contributing factors possibly affecting the change of anterior chamber angle parameters.

\section{Results}

No post-laser complications were found. The LV and LT did not change significantly 1 week after LPI. AOD500, AOD500 area, TISA500, TISA500 volume, TIA500, ACV and ACD increased significantly after LPI. There was significant decrease in ITC index and ITC area. LT was positively correlated to the change of ITC index $(r=0.411, p=0.002)$ and ITC area $(r=0.384, p=0.005)$. ACD was negatively correlated to the change of ITC index $(r=0.292, p=0.036)$.

\section{Conclusions}

The anterior segment architecture significantly changed after LPI in PAC spectrum eyes. Crystalline lens measurements remained unchanged before and after LPI. AS-OCT can be used to follow anterior chamber parameter changes in PAC spectrum eyes. Pre-treatment LT and ACD affect the therapeutic effect of LPI.

\section{Background}

Glaucoma is the second most common cause of blindness worldwide. Primary angle closure glaucoma (PACG) accounts for $25 \%$ of all kinds of glaucoma globally. It is the most common type of glaucoma among Chinese descent [1]. When the patients are found to have appositional or synechial closure of the anterior chamber angle, but with no visual field defect or glaucomatous optic nerve damage, they can be 
diagnosed as primary angle closure (PAC). It is an intermediate stage of the progression to PACG. Laser peripheral iridotomy (LPI) has been regarded as the primary choice to treat PAC by relieving pupillary block (PB) and widening the anterior chamber angle [2, 3]. However LPI is not sufficient to control IOP or prevent angle-closure progression [4]. The Academy of American Ophthalmology even demonstrated the superiority of cataract surgery over LPI in acute PAC [5]. Considering health economy, individual income and opportunity cost, widespread prophylactic LPI for PAC suspects is not recommended.

We often use gonioscopy, ultrasound biomicroscopy (UBM) or anterior segment optical coherence tomography (AS-OCT) to characterize LPI-induced anatomical changes. Gonioscopy, a subjective examination, doesn't possess the ability to accurately measure anterior chamber parameters. Compared with AS-OCT, UBM is a contact and time-consuming imaging modality with supine position required during examination. So AS-OCT is widely used to obtain high-resolution images of anterior chamber [6]. It has undergone several generations of innovation from time-domain OCT (TD-OCT), spectral-domain OCT (SD-OCT) to the swept-source OCT (SS-OCT). The CASIA2 SS-AS-OCT, with a high scan speed, provides a higher sensitivity for depth and higher resolution pictures. It has made it possible to obtain parameter data from cornea to posterior lens in one image just in a few seconds.

In the current study, we compared the anterior chamber, anterior chamber angle and lens parameters before and after LPI, aiming to demonstrate the safety and availability of LPI in PAC eyes and to examine baseline parameters associated with the effectiveness of LPI in Chinese population.

\section{Methods}

\section{Study design and patients enrollment}

This is a prospective, hospital-based, observational study that was approved by the Ethics Committee of Tianjin Medical University Eye Hospital [202KY(L)-52]. The study enrolled 28 patients (52 eyes), who underwent LPI for PAC state at the glaucoma clinic of Tianjin Medical University Eye Hospital in April 2020. The informed consent was signed before entering the study.

All enrolled eyes fulfilled the diagnosis criteria of PAC: 1. Narrow angles were found by gonioscopy (at least $180^{\circ}$ of trabecular meshwork was invisible, Spaeth grade A or B); 2 . One or more IOP lowering drugs were used or peripheral anterior synechiae (PAS) was found; 3 . No glaucomatous damage of the optic nerve was found (visual field was normal and vertical cup-to-disc ratio was no more than 0.6 ). The exclusion criteria were as follows: 1 . subjects unable to tolerate LPI treatment, for example, those with severe systemic diseases; 2 . subjects unable to cooperate well with the conductor to receive all the examinations; 3 . subjects not suitable for gonioscopy, for example, those with infectious conjunctivitis; 4 . eyes with apparent ocular diseases except for cataract; 5 . eyes with a history of laser procedure or intraocular surgery; 6 . eyes diagnosed as secondary angle closure; 7 . eyes with acute PAC or other conditions which needed surgery intervention. 
All participants underwent baseline ophthalmic examination including slit-lamp biomicroscopy, nonmydriatic fundus examination, gonioscopy, Goldmann applanation tonometer, visual field test. Slit-lamp biomicroscopy was done by a trained ophthalmologist to exclude subjects with other ocular diseases or abnormal cup-to-disc ratio. Static and dynamic gonioscopy were performed by an experienced gonioscopist to assess the condition of anterior chamber angle, to diagnose PAC and to identify the presence of PAS. The angle widths were graded using the Spaeth gonioscopic grading scale: A. open to Schwalbe's line; B. open to anterior trabecular meshwork; C. open to posterior trabecular meshwork; D. open to scleral spur; E. open to ciliary body band in each of 4 quadrants [5]. Visual field tests were done with the 24-2 pattern on the Humphrey Field Analyzer (Carl Zeiss Meditec, Dublin, CA). To avoid learning effect, the second reliable visual field result was accepted as the final one. Visual field results were considered abnormal if one of the following criteria was met: having a glaucoma hemifield test "outside normal limits", or pattern standard deviation (PSD) at a $\mathrm{P}<5 \%$ level, or a cluster of three non-edge locations worse than a $\mathrm{P}$ level of $5 \%$ with at least one worse than a $\mathrm{P}$ level of $1 \%$ on the pattern deviation plot (PDP) [8].

LPI was performed after all baseline examination being finished. Before LPI, miotic drops were given four times in half an hour. IOP was measured again 20 minutes after LPI. Prednisolone acetate and pranoprofen eye drops were administered 4 times a day for a week. IOP lowering drugs used by the patients remained unchanged before and after LPI. AS-OCT were performed before and 1 week after LPI for each subject. IOP was measured again 1 week after LPI.

\section{Image acquisition and parameter measurement}

AS-OCT was performed by an experienced ophthalmic imaging technician. All subjects underwent ASOCT (Tomey CASIA2) under ambient room lighting conditions. The CASIA2 uses a $1310 \mathrm{~nm}$ swept-source laser wavelength at a frequency of 0.3 seconds. With a high scan speed, it produces 12 images from 12 different angles in less than 2 seconds. Three consecutive scans were performed under "anterior chamber angle" mode. All patients had an AS-OCT scan performed by the same technician 1 week after LPI treatment.

The obtained images were analysed by the software provided by the manufacturer. This build-in software can automatically analyse the anterior segment structures and provide measurement results after scleral spurs (SSs) were marked. The SS was defined as the inward protrusion of the sclera where a change in curvature of the corneoscleral junction was observed. A trained technician who was masked to the gonioscopic grading marked SSs according to the rule. The analysed parameters included average angle opening distance (AOD500, defined as AOD at 500 $\mu \mathrm{m}$ from the marked SSs), AOD500 area (defined as the area formed by $360^{\circ}$ AOD500), average trabecular iris space area (TISA500, defined as the area formed by the following four points: trabecular meshwork at $500 \mu \mathrm{m}$ from the marked SSs, angle recess, intersection of trabecular meshwork at $500 \mu \mathrm{m}$ from the marked SSs perpendicular line and iris anterior surface, intersection of angle recess perpendicular line and iris anterior surface), TISA500 volume, (defined as the volume formed by $360^{\circ}$ TISA500), trabecular iris angle (TIA500, defined as the angle 
formed by trabecular meshwork at 500 $\mu \mathrm{m}$ from the marked SSs, angle recess and intersection of trabecular meshwork at 500 $\mathrm{m}$ from the marked SSs perpendicular line and iris anterior surface), iridotrabecular contact index (ITCl, defined as the percentage of iridotrabecular contact areas to all measured areas), ITC area, anterior chamber volume (ACV), anterior chamber depth (ACD), lens vault (LV, defined as the distance between the intersection of perpendicular bisector of SSs connection and lens and the midpoint of SSs connection) and lens thickness (LT).

\section{Statistical analysis}

Statistical analysis was performed by SPSS (Version 22). Continuous data were presented as mean \pm SD. Parameter variable before and after LPI analysis was performed using paired $t$-test. $P<0.05$ was considered statistically significant. Correlation between parameter variables was evaluated by the Pearson correlation analysis. The strength of the association was designated as no correlation for $0.0<r$ $<0.2$, weak correlation for $0.2<r<0.4$, moderate correlation for $0.4<r<0.6$, and strong correlation for 0.6 $<\mathrm{r}<1.0$.

\section{Results}

Fifty-two eyes of 28 PAC patients that qualified for the initial inclusion criteria were included in our study. Characteristics of the enrolled subjects at baseline were shown as follows. Among 28 patients 9 were male ( 16 eyes). The mean age of the subjects was $63.65 \pm 6.04$ years old with the range of 54 years old to 74 years old. The mean IOP pre-LPI was $17.35 \pm 3.61 \mathrm{mmHg}$. None of the patients had experienced postLPI complications such as persistent uveitis, persistent and serious IOP elevation or persistent pain.

There was a statistically significant increase in AOD500, AOD500 area, TISA500, TISA500 volume, TIA500, ACV and ACD before and 1 week after LPI. ITCI and ITC area decreased significantly post-LPI. There was no statistical significance in LV and LT before and 1 week after LPI. IOP increased significantly 15 mins after $\mathrm{LPI}$, while had a small but significant decrease 1 week after LPI. The results were shown in Table 1.

Table1. Anterior chamber and angle parameters measured by AS-OCT before and 1 week after LPI 


\begin{tabular}{|lll|}
\hline & Pre-LPI & One week post-LPI \\
\hline AOD500 $(\mathrm{mm})$ & $0.125 \pm 0.080$ & $0.257 \pm 0.106$ \\
\hline AOD500 area $\left(\mathrm{mm}^{2}\right)$ & $3.978 \pm 2.515$ & $7.942 \pm 3.336$ \\
\hline TISA500 $\left(\mathrm{mm}^{2}\right)$ & $0.045 \pm 0.031$ & $0.110 \pm 0.129$ \\
\hline TISA500 volume $\left(\mathrm{mm}^{3}\right)$ & $1.594 \pm 1.162$ & $3.163 \pm 1.344$ \\
\hline TIA500 $\left(^{\circ}\right)$ & $12.998 \pm 7.980$ & $24.831 \pm 9.476$ \\
\hline ITC index $(\%)$ & $41.440 \pm 29.468$ & $16.206 \pm 17.266$ \\
\hline ITC area $\left(\mathrm{mm}^{2}\right)$ & $6.399 \pm 6.119$ & $1.739 \pm 2.977$ \\
\hline ACD $(\mathrm{mm})$ & $1.818 \pm 0.262$ & $1.859 \pm 0.239$ \\
\hline ACV $\left(\mathrm{mm}{ }^{3}\right)$ & $68.946 \pm 16.112$ & $85.055 \pm 14.711$ \\
\hline LT $(\mathrm{mm})$ & $5.041 \pm 0.288$ & $5.007 \pm 0.290$ \\
\hline LV $(\mathrm{mm})$ & $0.898 \pm 0.333$ & $0.884 \pm 0.253$ \\
\hline
\end{tabular}

Scatterplots of LT and the change of ITCI, LT and the change of ITC area, LT and ACD, ACD and the change of ITCI were shown in Figure 1. There was moderate positive correlation between LT and the change of ITCI 1 week after LPI $(r=0.411, p=0.002)$ (Fig. 1A). There was weak positive correlation between LT and the change of ITC area 1 week after LPI $(r=0.384, p=0.005)$ (Fig. 1B). The negative correlation between LT and ACD was moderate $(r=-0.471, p=0.000)$ (Fig. 1C). There was weak positive correlation between ACD and the change of $\mathrm{ITCl}(r=0.292, p=0.036)$ (Fig. 1D). However, there was no correlation between ACD and the change of ITC area $(r=0.26, p=0.062)$.

\section{Discussion}

The current study utilized the novel commercially available SS-OCT to assess the change of anterior segment parameters after LPI in PAC eyes of Chinese descent. Compared with former types of AS-OCT, CASIA2, with high scanning speed and its built-in program, made the measurement of the average values of $360^{\circ}$ anterior chamber parameters in a few seconds possible. Similar with former studies, our results revealed that most anterior chamber parameters, such as AOD, TISA, TIA, ACD and ACV changed after $\mathrm{LPI}$, demonstrating the therapeutic effect of LPI $[9,10]$.

We also found more convincing data that ITCI and ITC area also changed.

In order to explore the pathogenesis of PAC and the effects of $\mathrm{LPI}$, we analyzed the correlation between biological indexes of anterior segments. It has been reported that ACD and LT can be regarded as predictable factors in the occurance of PACG $[11,12]$. The progressive decrease in ACD is age-related 
change over time. It will lead to PB, which is considered as the key element in the pathogenesis of PAC [13]. Moreover, the ITC index, as an indicator of the stages of PAC, was reported to be positively correlated with LT [14]. We found that the change of ITCl correlated with LT and ACD, which has not been reported before. Thick lens and shallow anterior chamber cobntribute to the occurance of PB. LPI is often performed to alleviate PB. So we think PAC eyes with thicker lens and shallower anterior chamber are more suitable for LPI treatment.

With good intra-device measurement reproducibility and repeatability [15], the novel commercially available SS-OCT, Tomey CASIA2 AS-OCT offers reliable quantitative measurements of anterior segment structures. Formerly used standard anterior segment OCT measures angle parameters in a single section. SS-OCT allows 3-dimensional measurements of angle parameters, such as the circumferential ITCI, ITC area and AOD area. Previously, we often used UBM to quantitatively assess angle parameters. One advantage superior over AS-OCT is that the ciliary body can be shown by UBM. However, ciliary bodies cannot be shown or assessed by AS-OCT because of blocking of the light through by the iris. AS-OCT still possesses some advantages over UBM. Higher degree of resolution, higher scan speed and the noncontact feature made the examination more comfortable, faster and safer for the patients. It is easier to learn and operate for the ophthalmic imaging technician. With a high scan speed of 50000 A-scans per second, it takes only $0.016 \mathrm{~s}$ to capture a single cross-sectional image, making the $360^{\circ}$ measurement of anterior chamber angle parameters done in just 2.4 seconds [16]. Considering the limitation of previously used instruments, most former studies only assessed the change of nasal and/or temporal anterior chamber angle parameters $[9,17]$. We measured average parameters around $360^{\circ}$ anterior segment using the built-in program in the current study, which provided more reliable and persuasive data.

LPI was widely used as a preventative treatment to PAC suspects and used to treat PAC and PACG patients without doubts before [18]. However, a large randomized trial conducted in China has demonstrated the limited effect of prophylactic LPI in PAC suspects in the prevention of vision threatening diseases occurrence [19]. EAGLE (Effectiveness of Early Lens Extraction for the Treatment of Primary Angle-Closure Glaucoma) study reported that early lens extraction is cost-effectively preferred over LPI in the management of PACG [20]. Another study reported that clear lens extraction resulted in a more significant effect in widening of the anterior chamber angle in PAC eyes without cataract than LPI [21]. Although these studies pointed out the benefits of clear lens extraction in PAC and PACG eyes, debates still exist. Considering the relatively short follow-up time, long-term benefits and safety of clear lens extraction should be further evaluated. It takes a long time and a lot of money to train qualified and experienced surgeons to do the surgery. They should master the procedure expertly and possess the ability to deal with all complications timely and properly [22]. Also, the cataract surgery costs much more than LPI. So currently, in developing countries, the role of LPI in the management of PAC eyes is still irreplaceable. Jiang et al. found that the drainage angle widened at 2 weeks after LPI in treated eyes [23]. He et al. also found LPI resulted in a significant increase in angle width in individuals with narrow angles [24]. Lin et al. reported that angle width changed at 2 weeks after LPI in narrow angle eyes without peripheral anterior synechia (PAS) [2]. So LPI was shown to be effective in treating PAC spectrum diseases in Chinese population based studies. 
Due to the wide use of $\mathrm{LPI}$, some research focused on finding the baseline factors possibly influencing the outcome of it. A New Zealand research found that pre-laser greater angle closure and less anterior iris bowing predicted the failure of LPI [25]. Another study reported that in South Indian population with PACS or PAC/ PACG, greater postoperative angle widening was associated with greater baseline PB, such as shorter AOD750, shorter axial length and greater lens vault [10]. PAS also influence the successful rate of LPI [2]. Baskaran et al. found that greater iris volume and higher IOP are risk factors for residual angle closure after LPI [26]. In the current study, we use the change of ITCI and ITC area before and one week after LPI to evaluate the short-term effect of it. Interestingly, we find LT and ACD correlates with the change of ITCl, which is never reported previously.

This study has a few limitations. One is that the sample size is small. More subjects need to be enrolled to verify the current results. Additionally, the follow-up time is short. Our study only evaluates the shot-

term effect of LPI. Long-term side effects and the effectiveness should be studied further. Finally, only one trained technician was responsible for marking SSs. Factors such as light conditions and the time of IOP measurement are not strictly controlled, which might increase the deviation of the parameters.

\section{Conclusions}

Our study describes the average parameters around $360^{\circ}$ anterior segment changes before and shortterm after LPI. Anterior chamber angle widens and ITC area decreases 1 week after LPI. When evaluating baseline parameters potentially affecting ITC change, we find that $L T$ is positively correlated with it. ACD is negatively correlated with it. Our results prove the beneficial effect of LPI in PAC eyes by AS-OCT. PAC patients with thicker lens and shallower anterior chamber respond better to LPI treatment. Novel SS-OCT is a better choice for anterior segment parameter measurement in clinic.

\section{Declarations}

Ethics approval and consent to participate: The study met the tenets of the Declaration of Helsinki and was approved by the Ethics Committee of Tianjin Medical University Eye Hospital [202KY(L)-52]. Written informed consent was obtained from all participants. All methods were carried in line with ethics approval.

Consent for publication: Not applicable.

Available of data and meterials: The datasets used and analyzed during the current study are available from the corresponding author on reasonable request.

Competing interests: The authors declare that they have no competing interests.

Funding: This research was supported by National Natural Science Foundation of China (grant number 81800825) and Tianjin Clinical Key Discipline Project (TJLCZDXKM011). 
Author's contributions: Conceptualization, X.X.; methodology, B.Y. and K.W.; data curation, B.Y.; writingoriginal draft preparation, B.Y. and K.W.; writing-review and editing, X.X.; supervision, X.X.; funding acquisition, X.X. and B.Y. All authors have read and agreed to the published version of the manuscript.

Acknowledgements: None.

\section{References}

1. Liang Y, Friedman DS, Zhou Q, Yang XH, Sun LP et al. Prevalence and characteristics of primary angle-closure diseases in a rural adult Chinese population: the Handan Eye Study. Investigative ophthalmology \& visual science. 2011;52(12):8672-9.

2. Lin Z, Liang Y, Wang N, Li S, Mou D et al. Peripheral anterior synechia reduce extent of angle widening after laser peripheral iridotomy in eyes with primary angle closure. J Glaucoma. 2013;22(5):374-9.

3. Robin AL, Pollack IP. Argon laser peripheral iridotomies in the treatment of primary angle closure glaucoma. Long-term follow-up. Arch Ophthalmol. 1982;100(6):919-23.

4. Aung T, Ang LP, Chan SP, Chew PT. Acute primary angle-closure: long-term intraocular pressure outcome in Asian eyes. Am J Ophthalmol. 2001;131(1):7-12.

5. Radhakrishnan S, Chen PP, Junk AK, Nouri-Mahdavi K, Chen TC. Laser Peripheral Iridotomy in Primary Angle Closure: A Report by the American Academy of Ophthalmology. Ophthalmology. 2018;125(7):1110-20.

6. Leung CK, Weinreb RN. Anterior chamber angle imaging with optical coherence tomography. Eye (Lond). 2011;25(3):261-7.

7. Spaeth GL. The normal development of the human anterior chamber angle: a new system of descriptive grading. Trans Ophthalmol Soc U K. 1971;91:709-39.

8. Hu R, Wang C, Racette L. Comparison of matrix frequency-doubling technology perimetry and standard automated perimetry in monitoring the development of visual field defects for glaucoma suspect eyes. PLoS ONE. 2017;12(5):e0178079.

9. Meduri E, Gillmann K, Bravetti GE, Niegowski LJ, Mermoud A et al. Iridocorneal Angle Assessment After Laser Iridotomy with Swept-source Optical Coherence Tomography. J Glaucoma. 2020.

10. Zebardast N, Kavitha S, Krishnamurthy P, Friedman DS, Nongpiur ME et al. Changes in Anterior Segment Morphology and Predictors of Angle Widening after Laser Iridotomy in South Indian Eyes. Ophthalmology. 2016;123(12):2519-26.

11. Saxena S, Agrawal PK, Pratap VB, Nath R. Anterior chamber depth and lens thickness in primary angle-closure glaucoma: a case-control study. Indian J Ophthalmol. 1993;41(2):71-3.

12. George R, Paul PG, Baskaran M, Ramesh SV, Raju P et al. Ocular biometry in occludable angles and angle closure glaucoma: a population based survey. The British journal of ophthalmology. 2003;87(4):399-402. 
13. Sun JH, Sung KR, Yun SC, Cheon MH, Tchah HW et al. Factors associated with anterior chamber narrowing with age: an optical coherence tomography study. Investigative ophthalmology \& visual science. 2012;53(6):2607-10.

14. Wang X, Chen X, Tang Y, Wang J, Chen $Y$ et al. Morphologic Features of Crystalline Lens in Patients with Primary Angle Closure Disease Observed by CASIA 2 Optical Coherence Tomography. Investigative ophthalmology \& visual science. 2020;61(5):40.

15. Xu BY, Mai DD, Penteado RC, Saunders L, Weinreb RN. Reproducibility and Agreement of Anterior Segment Parameter Measurements Obtained Using the CASIA2 and Spectralis OCT2 Optical Coherence Tomography Devices. J Glaucoma. 2017;26(11):974-9.

16. Shoji T, Kato $\mathrm{N}$, Ishikawa $\mathrm{S}$, Ibuki $\mathrm{H}$, Yamada $\mathrm{N}$ et al. In vivo crystalline lens measurements with novel swept-source optical coherent tomography: an investigation on variability of measurement. BMJ Open Ophthalmol. 2017;1(1):e000058.

17. Lee KS, Sung KR, Kang SY, Cho JW, Kim DY et al. Residual anterior chamber angle closure in narrowangle eyes following laser peripheral iridotomy: anterior segment optical coherence tomography quantitative study. Jpn J Ophthalmol. 2011;55(3):213-9.

18. Laser peripheral iridotomy for pupillary-block glaucoma. American Academy of Ophthalmology. Ophthalmology. 1994;101(10):1749-58.

19. He M, Jiang Y, Huang S, Chang DS, Munoz B et al. Laser peripheral iridotomy for the prevention of angle closure: a single-centre, randomised controlled trial. Lancet. 2019;393(10181):1609-18.

20. Azuara-Blanco A, Burr J, Ramsay C, Cooper D, Foster PJ et al. Effectiveness of early lens extraction for the treatment of primary angle-closure glaucoma (EAGLE): a randomised controlled trial. Lancet. 2016;388(10052):1389-97.

21. Melese E, Peterson JR, Feldman RM, Baker LA, Bell NP et al. Comparing Laser Peripheral Iridotomy to Cataract Extraction in Narrow Angle Eyes Using Anterior Segment Optical Coherence Tomography. PLoS ONE. 2016;11(9):e0162283.

22. Costa VP, Leung CKS, Kook MS, Lin SC, Global Glaucoma A. Clear lens extraction in eyes with primary angle closure and primary angle-closure glaucoma. Surv Ophthalmol. 2020;65(6):662-74.

23. Jiang $Y$, Chang DS, Zhu H, Khawaja AP, Aung $T$ et al. Longitudinal changes of angle configuration in primary angle-closure suspects: the Zhongshan Angle-Closure Prevention Trial. Ophthalmology. 2014;121(9):1699-705.

24. He M, Friedman DS, Ge J, Huang W, Jin C et al. Laser peripheral iridotomy in primary angle-closure suspects: biometric and gonioscopic outcomes: the Liwan Eye Study. Ophthalmology. 2007;114(3):494-500.

25. Ang G, Wells AP. Factors influencing laser peripheral iridotomy outcomes in white eyes: an anterior segment optical coherence tomography study. J Glaucoma. 2011;20(9):577-83.

26. Baskaran M, Yang E, Trikha S, Kumar RS, Wong HT et al. Residual Angle Closure One Year After Laser Peripheral Iridotomy in Primary Angle Closure Suspects. Am J Ophthalmol. 2017;183:111-7. 
Figures

\section{Figure 1}

Scatterplots between LT and the change of ITCI 1 week after LPI (A), LT and the change of ITC area 1 week after LPI (B), LT and ACD (C), ACD and the change of ITCI 1 week after LPI (D). 